\title{
Cesación tabáquica y promoción de la salud
}

\author{
Ana Mendoza Barbero \\ Farmacéutica comunitaria en Reus (Tarragona). Coordinadora del Grupo de Respiratorio de SEFAC.
}

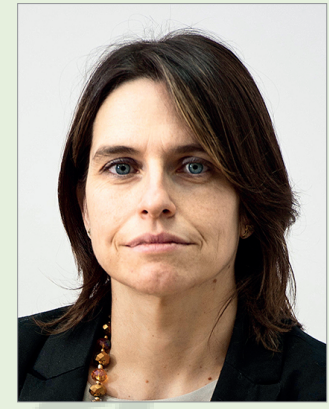

Ana Mendoza
El farmacéutico comunitario es un profesional sanitario cercano y accesible a la población. Su misión es la contribución en la mejora de la salud de los pacientes y el bienestar de la población debe ser la filosofía principal de la actividad farmacéutica. Para conseguir esto es necesario reforzar aspectos como el asesoramiento en la medicación y la provisión de servicios profesionales, de forma que abarquemos desde la resolución de consultas relacionadas con los medicamentos y la salud hasta la educación sanitaria.

Ya en el año 1946 la Organización Mundial de la Salud (OMS) definía la salud como el estado de completo bienestar físico, mental y social y no solamente la ausencia de enfermedad. Esta definición ha ido evolucionando y readaptándose. En 1986 en la Carta de Ottawa se define la promoción de la salud como el proceso que permite a las personas incrementar el control sobre su salud para mejorarla. La educación para la salud es el proceso que permite a las personas aumentar el control sobre determinantes de salud y así poderla mejorar.

La Federación Internacional Farmacéutica (FIP) ya habla de que los farmacéuticos deben ser reconocidos como profesionales sanitarios a quienes los pacientes pueden consultar sus problemas relacionados con la salud, y aquellos que no se puedan resolver en la farmacia se deriven al médico. Además, los farmacéuticos deben desarrollar y/o utilizar materiales educativos para programas de mantenimiento y promoción de la salud, y prevención de enfermedades dirigidos a un amplio rango de poblaciones de pacientes. Y lo más importante es que la FIP remarca que los farmacéuticos deben participar en actividades de prevención que fomenten la salud pública y prevengan enfermedades, particularmente en áreas como, por ejemplo, la cesación tabáquica (1).

Las farmacias comunitarias pueden desempeñar un papel fundamental en el abordaje del tabaquismo. La prevalencia del tabaquismo en España alcanza al 24 por ciento de la población según la última encuesta del Instituto Nacional de Estadística (INE), y ocasiona altas tasas de morbilidad (respiratoria, vascular, cáncer...) y de mortalidad (más de 55.000 muertes anuales). Además, el hecho de que el tabaquismo sea la principal causa de muerte evitable, convierten este problema de salud pública en un campo de especial interés para un abordaje multidisciplinar en el que el farmacéutico comunitario puede jugar un papel muy relevante por su proximidad a la población y conocimientos.

De cara a favorecer la intervención del farmacéutico comunitario en la cesación tabáquica podemos apoyarnos en tres premisas: 1) un 70 por ciento de los fumadores dice que le gustaría dejar el tabaco, 2) casi un 66 por ciento de los fumadores que recaen volverían a intentar el abandono en los siguientes 30 días y 3) los fumadores destacan el consejo sanitario para el abandono del tabaco como motivador para intentar dejar de fumar (2). Todos estos datos nos sugieren que muchos fumadores estarían interesados en dejar de fumar y que los profesionales sanitarios que estamos en contacto con estos pacientes podemos contribuir decisivamente a ello.

La intervención del farmacéutico comunitario en la cesación tabáquica puede tener gran importancia en la prevención y control de enfermedades relacionadas con el tabaco como puede ser el cáncer de pulmón, la insuficiencia cardíaca y la enfermedad cerebrovascular. Pero para optimizar el abordaje es necesaria la formación, la práctica y las guías consensuadas con sociedades médicas que nos avalen y guien en nuestras intervenciones.

Desde SEFAC se ha impulsado tanto la formación como la práctica a través del programa CESAR, en el que hasta ahora han participado más de 1.000 farmacéuticos comunitarios de toda España y 200 están ya registrando casos clínicos (más de 400 registros en la actualidad). Este programa pretende ofrecer a los farmacéuticos una capacitación integral para convertirse en especialistas en cesación tabáquica, siempre en colaboración con otros profesionales. Asimismo, CESAR ha servido para actualizar y reforzar la formación y entrenamiento del farmacéutico comunitario para la implantación y gestión efectiva de 
un servicio de cesación tabáquica en las farmacias comunitarias, capacitando profesionalmente y con la idea de conseguir la implantación de este servicio en las farmacias comunitarias.

Además, y con el fin de facilitar la prestación protocolizada de este servicio, se ha presentado recientemente el Documento de intervención en cesación tabáquica en farmacia comunitaria. Este documento es el primero de sus características que cuenta con el apoyo de las sociedades médicas de atención primaria Semergen, semFYC y SEMG, la Sociedad Española de Neumología y Cirugía Torácica (SEPAR) y la Sociedad Española de Especialistas en Tabaquismo (SEDET), además de haber sido prologada por el presidente del Comité Nacional para la Prevención del Tabaquismo (CNPT). Este documento quiere ser una guía para sentar bases comunes de intervención en la farmacia comunitaria en cuestiones de tabaquismo (3).

El documento aporta información práctica para llevar a cabo el servicio de cesación tabáquica como, por ejemplo, los grupos diana a los que el consumo de tabaco les afecta especialmente bien por empeorar su enfermedad, por interferir en el tratamiento o por agravar efectos adversos (pacientes con patologías respiratorias, cardiovascular, con problemas dermatológicos, pacientes psiquiátricos, etc.). El texto también repasa aquellas situaciones que pueden facilitar la intervención en cesación tabáquica de los farmacéuticos, por ejemplo, en la indicación farmacéutica en síntomas menores respiratorios, en la medida de parámetros biológicos (presión arterial, colesterol, etc.), en consejo nutricional y durante el seguimiento farmacoterapéutico. Del mismo modo, se incluye información sobre las distintas estrategias y herramientas que puede emplear el farmacéutico comunitario al prestar este servicio (la entrevista clínica, test de Fagerström, etc.) e información sobre los tratamientos existentes (tanto farmacológicos como no farmacológicos) y los criterios de derivación al médico, entre otros muchos aspectos.

A todas estas iniciativas, y con vistas a impulsar aún más este servicio, SEFAC tiene previsto evaluar y cuantificar próximamente el impacto de la intervención farmacéutica en la cesación tabáquica, tanto a nivel sanitario como económico. Hasta el momento, y según los datos preliminares de las intervenciones realizadas en CESAR, alrededor del 46 por ciento de los pacientes que inician el servicio de cesación tabáquica en una farmacia comunitaria consiguen dejar de fumar a los seis meses.

Convirtamos pues nuestras farmacias en espacios de salud donde se puedan realizar campañas de sensibilización (por ejemplo, para la valoración de la edad pulmonar con el objetivo de motivar a aquellas personas fumadoras que todavía no se hayan planteado dejar de fumar) y preguntemos siempre a los usuarios de la farmacia sobre el hábito tabáquico. La detección de fumadores aumenta el porcentaje de actuación con el consiguiente aumento de las tasas de cesación gracias al asesoramiento.

La profesión farmacéutica ha evolucionado adquiriendo un mayor protagonismo en el ámbito asistencial. En ello ha tenido que ver mucho la atención farmacéutica. Demos un paso más e implantemos servicios que aporten valor añadido y respondan a las necesidades de la población.

\section{Referencias bibliográficas}

1. Federación Internacional Farmacéutica [Internet]. Buenas Prácticas en Farmacia. Directrices FIP/OMS sobre Buenas Prácticas en Farmacia: Estándares para la calidad de los servicios farmacéuticos. 2012. [Consultado 12/11/ 2014]. Disponible en: https://www.fip.org/www/uploads/database_file. php?id=334ttable_id=

2. Fiore MC, Jaén CR, Baker TB, Bayley WWC, Benowitz NL, Curry SJ, et al. Treating Tobacco Use and Dependence: 2008 Update. Clinical Practice Guideline. Rockville, MD: U.S. Departament of Health and Human Services. Public Health Service. May 2008. Traducción al Español: Jiménez Ruiz CA, Jaén Cr (Coordinadores de la traducción). Guía de tratamiento del tabaquismo. Barcelona: Sociedad Española de Neumología y Cirugía Torácica; 2010.

3. Gómez Martínez JC [Coordinador]. Documento de Intervención en Cesación Tabáquica en la Farmacia Comunitaria. Madrid: Sociedad Española de Farmacia Familiar y Comunitaria; 2014. ISBN: 978-84-616-9874-5 (http://www.sefac.org/contenido/ documento-de-intervenci\%C3\%B3n-en-cesaci\%C3\%B3n-tab\%C3\%A1quica-en-la-farmacia-comunitaria) 